\title{
Quantum pumping in graphene
}

\author{
E. Prada, P. San-Jose, and H. Schomerus \\ Department of Physics, Lancaster University, Lancaster, LA1 4YB, United Kingdom
}

(Dated: December 15, 2009)

\begin{abstract}
We show that graphene-based quantum pumps can tap into evanescent modes, which penetrate deeply into the device as a consequence of Klein tunneling. The evanescent modes dominate pumping at the Dirac point, and give rise to a universal response under weak driving for short and wide pumps, in close analogy to their role for the minimal conductivity in ballistic transport. In contrast, evanescent modes contribute negligibly to normal pumps. Our findings add a new incentive for the exploration of graphene-based nanoelectronic devices.
\end{abstract}

\section{INTRODUCTION}

Quantum pumps transfer electrons between two reservoirs by externally varying their scattering properties over time. This concept has attracted much attention since its inception, due in part to its promise for practical applications in nanoelectronics ${ }^{122}$ and for the definition of a current standard, $\sqrt[3]{5}$ but also because of its elegant theoretical description in terms of the geometry of the control parameter manifold ${ }^{[6] 9}$

Efficient quantum pumping requires strong but energydependent coupling of the pump to the reservoirs. In normal systems, pumping is therefore constrained to propagating modes, while the poorly coupled evanescent modes decay rapidly away from the contacts and therefore cannot contribute to the pumped charge. Here, we show that the discovery of graphene-based material ${ }^{10}$ calls for a revision of such common concepts in quantum pumping. In graphene, the low-energy charge carriers are described by a massless Dirac equation, 11 and the unique feature of chirality suppresses backscattering at interfaces, resulting in the so-called Klein paradox by which charge carriers are difficult to confine ${ }^{12 \mid 14}$ This seems to inhibit the prospects of quantum pumping - unless one properly accounts for the effects of chirality on the evanescent modes. These effects so far have been explored only for stationary ballistic transport, where evanescent chiral electrons manifest themselves in macroscopic quantum tunneling close to the Dirac point of charge-neutral graphene. $\frac{15 / 16}{16}$ We show that the scattering of these evanescent modes is sufficiently energy-dependent so that they contribute significantly to quantum pumping. Close to the Dirac point, they deliver the dominant contribution to the pumped charge, which can be characterized by a universal dimensionless pumping efficiency.

We start this paper in Sec. II with a description of the employed model (a quantum pump driven by two electrostatic gates) and method (the scattering approach to adiabatic quantum pumping). Our results are presented in Sec. III. The concluding remarks in Sec. IV]also contain a discussion of practical advantages of graphene-based quantum pumps.

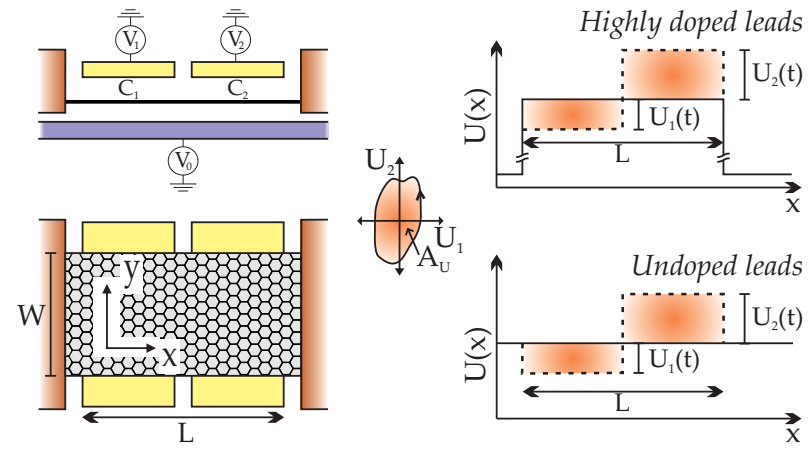

FIG. 1. (Color online) Left panels: Illustration of a graphene quantum pump with highly doped (metallic) contacts. Two gates at voltage $V_{1}(t)$ and $V_{2}(t)$ control the time-dependent onsite energy $U_{1}(t)$ and $U_{2}(t)$ in the graphene flake over a pumping cycle. This induces a charge transport between two contact electrodes, separated by a distance $L$. The working point of the device can be further controlled by an additional back gate with voltage $V_{0}$. Right panels: Snapshots of the onsite potential along the quantum pump, for highly doped (metallic) contacts (top) and undoped contacts (bottom). The solid base line shows the potential at the working point. We show that evanescent modes induced by metallic contacts enable finite charge pumping at vanishing nominal charge-carrier density, in striking contrast to the case of normal pumps where the nanoribbon is replaced by a normal conductor.

\section{MODEL AND METHOD}

In order to characterize the unique features of quantum pumping in graphene, we compare the behavior of four different setups: graphene pumps with either heavily doped contacts or undoped contacts are contrasted with normal-conductor pumps with these two types of contacts. In the absence of driving (i.e., at the working point of the pump), the systems with undoped contacts have a uniform carrier density, equal in both the leads and in the central pumping region. In contrast, the charge carrier density in heavily doped contacts is very large; this can be realized by metallic contacts $\frac{17}{5}$. We assume that the charge carrier density in the system can be further uniformly shifted by a back gate covering both the pumping region and leads, thereby tuning the working 
point of the device. For heavily doped contacts, the backgate-induced change of the charge carrier density in the leads can be neglected. Since only the setup with highly doped contacts induces evanescent modes, this comparison allows to isolate the requirements for the successful deployment of such modes in quantum pumping.

In all of these four cases, we consider a standard pumping mechanism, which is shown for the example of a graphene pump with heavily doped (metallic) contacts in the left panels of Fig. 1] The pump is operated by a cyclic raising and lowering of the potential in two additional electrostatic gates, which modulate the charge carrier density in the two halves of the system due to their effect on the onsite potential $U$. This induces charge transfer between the two electronic reservoirs of the contacts. The bottom left panel shows a rotated view of the pump, which is further characterized by the width $W$ and length $L$ of the pumping region. The top right panel shows a snapshot of the onsite potential (dashed line) for the case of highly n-doped contacts (the solid line is the onsite potential at the working point). The bottom right panel shows a corresponding snapshot for undoped leads.

An elegant formulation of quantum pumping is afforded by the scattering approach 778 , which considers the time dependence of the scattering matrix $S(t)$. In the minimal case of adiabatic driving with two independent parameters $\boldsymbol{\xi}=\left\{\xi_{1}, \xi_{2}\right\}$ and single channel reservoirs, the charge transferred across the non-interacting scattering region reduces to an integral over the area enclosed by the driving path in two-dimensional (2D) parameter $\operatorname{space}^{18}$,

$$
\begin{aligned}
Q & =\int d \xi_{1} d \xi_{2} \partial_{\xi}^{2} Q(\boldsymbol{\xi}), \\
\partial_{\xi}^{2} Q & \equiv \frac{1}{2 \pi}\left(\frac{\partial T}{\partial \xi_{1}} \frac{\partial \phi}{\partial \xi_{2}}-\frac{\partial T}{\partial \xi_{2}} \frac{\partial \phi}{\partial \xi_{1}}\right) .
\end{aligned}
$$

The transmission probability $T$ and the scattering phase $\phi=\alpha-\beta$ above are determined by the scattering matrix

$$
S=\left(\begin{array}{ll}
r & t^{\prime} \\
t & r^{\prime}
\end{array}\right)=e^{i \gamma}\left(\begin{array}{cc}
\sqrt{1-T} e^{i \alpha} & -\sqrt{T} e^{i \beta} \\
\sqrt{T} e^{-i \beta} & \sqrt{1-T} e^{-i \alpha}
\end{array}\right),
$$

where $r\left(r^{\prime}\right)$ and $t\left(t^{\prime}\right)$ are reflection and transmission amplitudes for electrons arriving from the left (right) reservoir.

In a quasi one-dimensional setup with more than one channel, indexed by quantum number $q$, the total pumped charge will be a sum over channels $Q=\sum_{q} Q_{q}$ as long as they can be considered independent. This is the case for the quantum pump setup depicted in Fig. 1 . Across the width $W$ of the system, the onsite potential $U$ is $y$ independent, so that different scattering channels $q$ remain decoupled. We model this profile by two abrupt potential steps in the $x$ direction of equal length $L / 2$ and assume the two driving parameters $\boldsymbol{\xi}=\left\{U_{1}(t), U_{2}(t)\right\}$ have zero average, and maximum amplitudes $\delta U_{1}$ and $\delta U_{2}$, respectively. A back gate is used to control the carrier concentration in both pump and leads, which we parameterize by the average Fermi momentum $k_{F}$ in the pumping region. Undoped contacts have the same Fermi momentum $k_{F}$ as the pumping region, while heavily doped contacts have a much larger Fermi momentum, which can effectively be taken as infinite ${ }^{[19]}$ By employing the Dirac equation with negligible inter-valley scattering for graphene ${ }^{12}$, and an effective mass approximation for the normal system, this model allows us to compute the transmission probabilities and phases $T_{q}$ and $\phi_{q}$ by simple wave matching.

\section{RESULTS AND DISCUSSION}

The finite length $L$ of the pump provides a natural scale for the scattering problem. It fixes the energy scales $E_{L}^{G}=\hbar v_{0} / L$ (with Dirac velocity $v_{0}$ ) in the graphene case, and $E_{L}^{N}=\hbar^{2} /\left(2 m^{*} L^{2}\right)$ (with effective mass $m^{*}$ ) for the normal conductor, which are related to the level spacing of the isolated pump. These energies determine two possible driving regimes, depending on the maximum amplitude $\delta u_{i}$ of the dimensionless driving energies $u_{i} \equiv$ $U_{i} / E_{L}$. In the weak driving regime, $\delta u_{i} \ll 1$, the charge pumped in channel $q$ can be approximated by

$$
Q_{q} \approx \partial_{u}^{2} Q_{q}(0) \int d u_{1} d u_{2}=A_{u} \partial_{u}^{2} Q_{q}(0),
$$

where $A_{u} \sim \delta u_{1} \delta u_{2}$ is the small area enclosed in parameter space by the driving cycle, wherein $\partial_{u}^{2} Q_{q}\left(u_{1}, u_{2}\right)$ can be approximated by a constant. Away from this regime the integral in Eq. (1) has to be performed numerically.

For the graphene pump with heavily doped contacts, Eq. (2) yields

$$
\begin{aligned}
Q_{q}^{\mathrm{gr}-\infty} & = \pm A_{u} \frac{k_{F} L}{\pi} \frac{(q L)^{2}}{k_{x} L} \\
& \times \frac{\sin ^{2}\left(k_{x} L\right)\left[\sin \left(2 k_{x} L\right)-2 k_{x} L \cos \left(2 k_{x} L\right)\right]}{\left[\left(k_{x} L\right)^{2}+(q L)^{2} \sin ^{2}\left(2 k_{x} L\right)\right]^{2}} .
\end{aligned}
$$

Here the \pm sign denotes whether the pump is doped with electrons (plus) or holes, and $k_{x}=\sqrt{k_{F}^{2}-q^{2}}$ is the electron's momentum along the transport direction in the pumping region. Momentum $k_{x}$ is real for propagating modes $|q|<k_{F}$ and imaginary for modes $|q|>k_{F}$ that are evanescent in the pump. In contrast, a graphene pump with undoped leads has no incoming lead modes that become evanescent in the pump (in the limit $\delta u_{i} \ll 1$ ), since the Fermi momentum in the pump at the working point is identical to the Fermi momentum in the contacts. A graphene setup with undoped leads pumps a charge

$$
Q_{q}^{\mathrm{gr}-0}=A_{u} \frac{k_{F} L}{\pi} \frac{2(q L)^{2} \cos ^{2}\left(k_{x} L\right) \sin ^{3}\left(k_{x} L\right)}{\left(k_{x} L\right)^{4}},
$$

where $k_{x}$ is constrained to real values since $|q| \leq k_{F}$.

In both cases the pumped charge has a prefactor $A_{u} k_{F}$, indicating that pumping is proportional to the pump's 


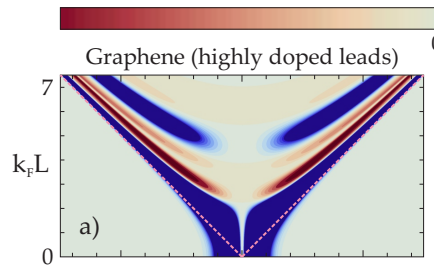

Graphene (undoped leads)

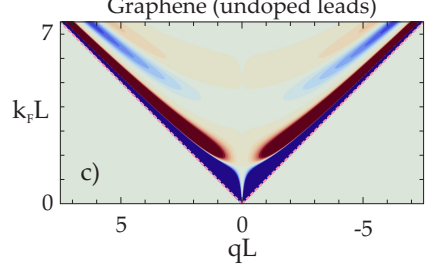

Normal conductor (undoped leads)

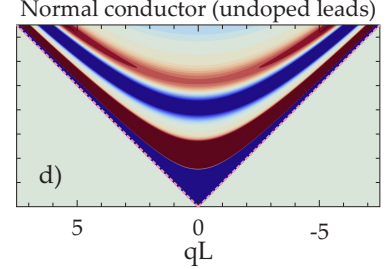

FIG. 2. (Color online) Momentum distribution of pumped charge per mode $\chi_{q}^{u}$ as a function of mode index $q$ for varying carrier concentration (parameterized by the Fermi momentum $\left.k_{F}\right)$. Blue and brown represent opposite directions of pumping (left to right or right to left). In graphene, the propagating mode with $q=0$ (normal incidence) cannot be pumped due to the Klein paradox. In the case of graphene with heavily doped leads, however, significant pumping is possible due to the contribution of the evanescent modes $\left(|q|>k_{F}\right.$, delineated by the dashed line), which dominate around the Dirac point $\left(k_{F}=0\right)$. The other pumps can only drive current through the propagating modes.

number $N_{p}=4 k_{F} W / \pi$ of propagating electrons at the Fermi energy and the dimensionless driving strength $A_{u}$. By factoring out these two quantities, we obtain the dimensionless pumping response

$$
\chi_{q}^{u} \equiv \frac{\partial_{u}^{2} Q_{q}}{N_{p}} \approx \frac{Q_{q}}{A_{u} N_{p}},
$$

which depends only on the system's scattering characteristics at a given energy. Summing over all incoming modes gives the fraction of (propagating) electrons in the pump that are pumped per cycle and per unit dimensionless driving strength $A_{u}$,

$$
\chi^{u}=\sum_{q} \chi_{q}^{u}=\frac{1}{N_{p}} \sum_{q} \partial_{u}^{2} Q_{q} .
$$

This defines the total dimensionless pumping response per mode. For short and wide pumps $(W \gg L)$, the sum over modes can be approximated by an integral.

Similar results can be derived for the normal pump,

$$
\begin{aligned}
Q_{q}^{\mathrm{n}} & =A_{u} 8 \frac{k_{F}^{2} K_{x}^{3}}{k_{x} L^{2}} \sin ^{2}\left(k_{x} L\right) \\
& \times \frac{2 k_{x} L\left(k_{x}^{2}-K_{x}^{2}\right) \cos \left(2 k_{x} L\right)+\left(k_{x}^{2}+K_{x}^{2}\right) \sin \left(2 k_{x} L\right)}{\left[4 k_{x}^{2} K_{x}^{2} \cos ^{2}\left(2 k_{x} L\right)+\left(k_{x}^{2}+K_{x}^{2}\right)^{2} \sin ^{2}\left(2 k_{x} L\right)\right]^{2}}
\end{aligned}
$$

Both the heavily doped contact $\left(Q_{q}^{\mathrm{n}-\infty}\right)$ and undoped contact $\left(Q_{q}^{\mathrm{n}-0}\right)$ normal cases can be derived from the above by taking the $x$ momentum in the leads $K_{x}$ to infinity or $k_{x}$ respectively.

In Fig. 2 we represent the pumping response $\chi_{q}^{u}$ of Eq. (5) as a function of $q$ and $k_{F}$ in the two graphene setups, and compare these with the results of the normal pumps. For graphene we only show results for electron carriers. If the leads are heavily doped the pumped current reverts sign when the carriers in the central pump region are changed from electrons to holes [cf. Eq. (3)]. In the case of undoped leads, the pumped current remains the same and only reverts sign if one also reverts the pumping cycle.

In all four panels, the dashed line delineates the threshold between evanescent and propagating modes. The most evident feature in the graphene pump with heavily doped leads [Fig. 2(a)] is the contribution of evanescent modes to pumping close to the Dirac point. This contribution is absent in the other three cases, in which only propagating electrons are pumped. The reason for this is the high transmission of evanescent modes in graphene, which can be attributed to chirality and the Klein paradox ${ }^{13}$. Unlike in the normal pump with heavily doped leads, chirality conservation at the contact enables evanescent electrons to populate the graphene pumping region for modes within a window of width $\Delta q \sim 1 / L$ around $q=0$. These evanescent modes contribute to pumping because they are sensitive to the onsite potentials $U_{i}$ and have a finite amplitude at both contacts, so that charge transfer between them is possible over a pumping cycle. The finite contribution of the evanescent modes in graphene is in striking contrast to the vanishing contribution of electrons in the propagating mode $q=0$ (normal incidence), for which the transmission $T_{q=0}=1$ is perfect at all energies because of the Klein paradoxthese modes are therefore insensitive to driving and cannot be pumped.

In a normal pump with heavily doped contacts [Fig. 2(b)], the large Fermi velocity mismatch suppresses the transparency of the contacts for all modes except those at resonant tunneling. This confinement creates sharply defined energy levels in the pump which are the origin of narrow regions of finite pumping, and results in a threshold $k_{F} L \simeq 1$ below which no pumping occurs. In particular, the contribution of evanescent modes to pumping is strongly suppressed (no contribution outside of the dashed line). Moreover, normal pumping in the limit of large lead doping is directed, meaning that for a given orientation of the driving cycle, the pumped current has the same sign for all energies.

For graphene and normal pumps with undoped leads [Fig. 2(c) and (d)], all incoming modes remain propagating in the pumping region. The main difference between graphene and normal pumps in this limit is the effect of the Klein paradox in the former, which suppresses pumping at $q=0$, just as in the case of graphene with doped leads. Both pumps are open, and consequently there is no energy threshold for pumping. The sign of the pumped current is energy dependent, which is a generic feature of open pumps (including graphene pump with doped leads). 


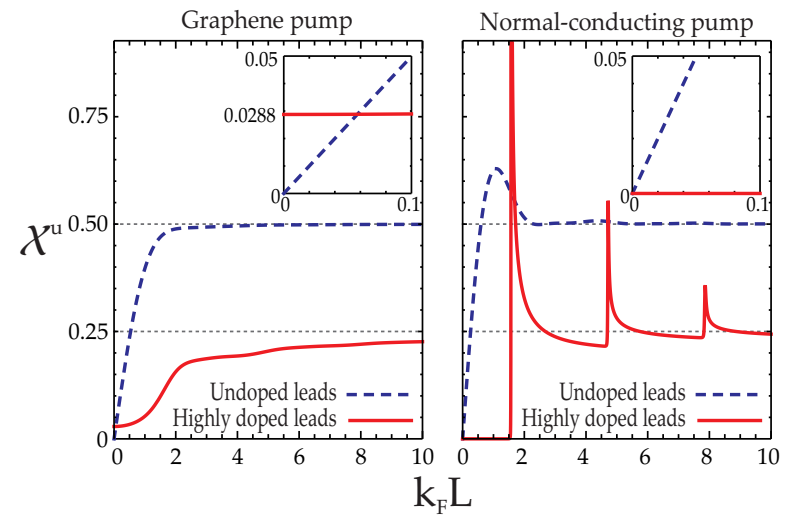

FIG. 3. (Color online) Total dimensionless response per mode $\chi^{u}$ for short and wide pumps $(W \gg L)$, as a function of the carrier concentration (parameterized by the Fermi momentum $k_{F}$ ). Left panel: graphene pumps. Right panel: Normal-conducting pumps. Solid lines correspond to pumps with highly doped leads (which tend to $1 / 4$ at $k_{F} L \gg 1$ ), while dashed lines correspond to pumps with undoped leads (which tend to $1 / 2)$. At the charge neutrality $\left(k_{F} L \rightarrow 0\right.$, see inset), evanescent modes allow for a finite charge transfer in the highly-doped-leads graphene pump, whose response saturates at a universal (sample-independent) value of 0.0288 .

In Fig. 3 we plot the total dimensionless pumping response $\chi^{u}\left(k_{F}\right)$ of Eq. (6) for the four types of pumps considered (left panel: graphene pumps; right panel: normal-conducting pumps). The regime of evanescent electron pumping in graphene with highly doped leads is visible as a saturation at $k_{F}=0$ (see the solid curve in the inset). For wide and short pumps $(W \gg L)$, this saturation value is sample independent and takes the dimensionless value

$$
\int_{0}^{\infty} d q \frac{\sinh ^{2}(q)[2 q \cosh (2 q)-\sinh (2 q)]}{\pi q^{3} \cosh ^{4}(2 q)}=0.0288
$$

which is the analogue of the minimal conductivity in the context of pumping 15/16]. In contrast, all other pumps have a vanishing pumping response at depletion. At energies $k_{F} \gtrsim 1 / L$, the pumping response rises to $1 / 2$ and $1 / 4$ in the cases of undoped and highly doped leads, respectively. The normal pump with highly doped leads, however, only operates above a finite carrier-concentration threshold corresponding to the position of the first resonant tunneling subband as mentioned above.

\section{CONCLUDING REMARKS}

In summary, we find that evanescent modes can contribute significantly to graphene-based quantum pump- ing, to the extent that they provide the dominant contribution to the pumped charge when the system is operated close to the charge-neutrality point. Our comparison to results for normal pumps reveals that this effect is intimately related to chirality and the Klein paradox, and therefore arises from the unique low-energy properties of charge carriers in graphene. For the case of short and wide pumps, the evanescent pumping regime is characterized by a sample-independent universal value of the dimensionless pumping response.

Practical considerations point towards additional advantages of graphene-based quantum pumps. Firstly, in a realistic experimental setup, the principal pump driving parameters are not the onsite energies $U_{i}$, but gate voltages $V_{i}$ (see Fig. 1) which manipulate the locally induced charge density $n_{i}(t)=V_{i}(t) C_{i}$ under each gate depending on the capacitances $C_{i}$. The onsite energies then follow from the difference of the Fermi energy and the local position of the Dirac point. Neglecting details of the screening, for graphene $n_{i}=W u_{i}^{2} /(\pi L)$, whilst for the normal case $n_{i}=W u_{i} /(2 \pi L)$ (in the case of graphene with highly doped leads, a precise modeling would also have to take care of charge carriers populating the evanescent modes). In terms of these charge densities, the relevant response function is

$$
\chi^{n}=\chi^{u} \operatorname{det}\left(\frac{\partial u_{i}}{\partial n_{j}}\right)
$$

involving the Jacobian between the $u$ and the $n$ variables. Due to the divergence $\partial u_{i} / \partial n_{i} \propto n_{i}^{-1 / 2}$ close to the Dirac point, the experimental pumping response $\chi^{n}$ for graphene pumps is expected to rapidly increase as one approaches charge neutrality, while it vanishes for normal pumps. Secondly, graphene-based quantum pumping promises to display an enhanced robustness against thermal effects. Thermal smearing of the pumping response 20 occurs at temperatures of order $E_{L} / k_{B}$, which are considerably higher in graphene than in normal pumps (this is also one of the reasons for the temperature robustness of other transport effects in graphene $e^{21}$ ). For the same reason, mechanisms limiting the pumping frequency are expected to be less stringent in graphene than in normal pumps. These considerations, together with the long coherence length and high mobility in graphene, lead us to believe that graphene-based quantum pumps have good chances to become an experimental reality.

We acknowledge support from the European Commission, Marie Curie Excellence Grant MEXT-CT-2005023778 .

1 M. Switkes, C. Marcus, K. Campman, and A. Gossard,

${ }^{2}$ S. K. Watson, R. M. Potok, C. M. Marcus, and V. UmanScience 283, 1905 (Mar. 1999). 
sky, Phys. Rev. Lett. 91, 258301 (Dec. 2003).

3 S. J. Wright, M. D. Blumenthal, M. Pepper, D. Anderson, G. A. C. Jones, C. A. Nicoll, and D. A. Ritchie, Phys. Rev. B, 113303(2009).

4 J. Pekola, J. Vartiainen, M. Möttönen, O. Saira, M. Meschke, and D. Averin, Nature Physics 4, 120 (2007).

${ }^{5}$ L. P. Kouwenhoven, A. T. Johnson, N. C. van der Vaart, C. J. P. M. Harmans, and C. T. Foxon, Phys. Rev. Lett 67, 1626 (1991).

6 M. Büttiker, H. Thomas, and A. Pretre, Z. Phys. B 94, 133 (April 1994).

7 P. W. Brouwer, Phys. Rev. B 58, R10135 (1998).

${ }^{8}$ Y. Makhlin and A. D. Mirlin, Phys. Rev. Lett. 87, 276803 (2001).

9 Y. Aharonov and J. Anandan, Phys. Rev. Lett. 58, 1593 (1987).

10 K. Novoselov, A. Geim, S. Morozov, D. Jiang, Y. Zhang, S. Dubonos, I. Grigorieva, and A. Firsov, Science 306, 666 (2004).

11 K. Novoselov, A. Geim, S. Morozov, D. Jiang, M. Katsnelson, I. Grigorieva, S. Dubonos, and A. Firsov, Nature 438, 197 (2005).

12 A. H. C. Neto, N. M. R. Peres, K. S. Novoselov, and A. K. Geim, Rev. Mod. Phys. 81, 109 (2009).

13 M. Katsnelson, K. Novoselov, and A. Geim, Nature Physics 2, 620 (2006).

14 C. W. J. Beenakker, Rev. Mod. Phys. 80, 1337 (2008).

15 M. Katsnelson, Eur. Phys. J. B 51, 157 (2006).

16 J. Tworzydlo, B. Trauzettel, M. Titov, A. Rycerz, and C. W. J. Beenakker, Phys. Rev. Lett. 96, 246802 (2006).

17 H. Schomerus, Phys. Rev. B 76, 045433 (2007).

18 Throughout this work, the term charge refers to the number of electrons.

19 All our results therefore do not feature an additional Fermi momentum for the leads.

20 M. G. Vavilov, V. Ambegaokar, and I. L. Aleiner, Phys. Rev. B 63, 195313 (2001).

${ }^{21}$ K. Novoselov, E. McCann, S. Morozov, V. Fal'ko, M. Katsnelson, U. Zeitler, D. Jiang, F. Schedin, and A. Geim, Nat. Phys. 2, 177 (2006). 